\title{
Mesenchymal Stem Cells in Bone Regeneration
}

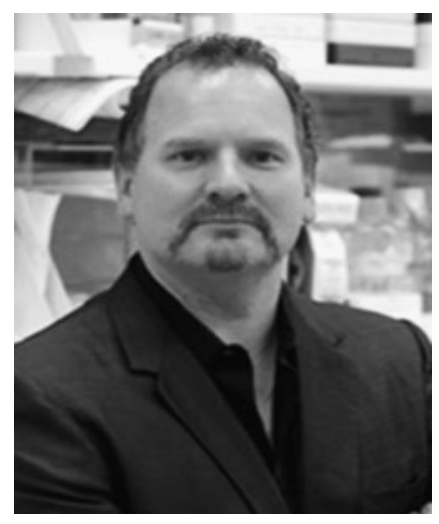

Kurt D. Hankenson, DVM, PhD

Submitted for publication February 8, 2013. ${ }^{*}$ Correspondence: Dept. of Clinical Studies, New Bolton Center, Room 145 Myrin Bldg., 382 W. Street Rd., Kennett Square, PA 19348 (e-mail: kdhank@vet.upenn.edu).

\section{Abbreviations and Acronyms \\ $\alpha \mathrm{SMA}=$ alpha smooth muscle actin \\ A-MSC $=$ adipose-derived mesenchymal stem cells \\ $A B G=$ autologous bone graft \\ $A L P=$ alkaline phosphatase \\ $\mathrm{B}-\mathrm{MSC}=$ bone-derived mesen- chymal stem cells \\ $\mathrm{BM}-\mathrm{MSC}=$ bone marrow - derived mesenchymal stem cells $\mathrm{BMP}=$ bone morphogenetic protein \\ $\mathrm{BMT}=$ bone marrow transplant \\ $\mathrm{CXCR4}=\mathrm{C}-\mathrm{X}-\mathrm{C}$ chemokine \\ receptor type 4 \\ Dkk1 $=$ dickkopf 1 \\ $\mathrm{DBM}=$ demineralized bone matrix \\ $\mathrm{GFP}=$ green fluorescent protein \\ (continued)}

\author{
M. Noelle Knight ${ }^{1,2}$ and Kurt D. Hankenson ${ }^{2,3, *}$ \\ ${ }^{1}$ Veterinary Medical Scientist Training Program and ${ }^{2}$ Department of Clinical Studies, School of Veterinary Medicine \\ ${ }^{3}$ Department of Orthopaedic Surgery, Perelman School of Medicine; University of Pennsylvania, Philadelphia, \\ Pennsylvania.
}

Significance: Mesenchymal stem cells (MSCs) play a key role in fracture repair by differentiating to become bone-forming osteoblasts and cartilageforming chondrocytes. Cartilage then serves as a template for additional bone formation through the process of endochondral ossification.

Recent Advances: Endogenous MSCs that contribute to healing are primarily derived from the periosteum, endosteum, and marrow cavity, but also may be contributed from the overlying muscle or through systemic circulation, depending on the type of injury. A variety of growth factor signaling pathways, including BMP, Wnt, and Notch signaling, influence MSC proliferation and differentiation. These MSCs can be therapeutically manipulated to promote differentiation. Furthermore, MSCs can be harvested, cultivated, and delivered to promote bone healing.

Critical Issues: Pharmacologically manipulating the number and differentiation capacity of endogenous MSCs is one potential therapeutic approach to improve healing; however, ideal agents to influence signaling pathways need to be developed and additional therapeutics that activate endogenous MSCs are needed. Whether isolated and purified, MSCs participate directly in the healing process or serve a bystander effect and indirectly influence healing is not well defined.

Future Directions: Studies must focus on better understanding the regulation of endogenous MSCs durings fracture healing. This will reveal novel molecules and pathways to therapeutically target. Similarly, while animal models have demonstrated efficacy in the delivery of MSCs to promote healing, more research is needed to understand ideal donor cells, cultivation methods, and delivery before stem cell therapy approaches can be utilized to repair bone.

\section{SCOPE AND SIGNIFICANCE}

BONE IS A UNIQUE TISSUE that completely regenerates, rather than healing with a scar after injury like many other tissues. ${ }^{1}$ The beginning stages of fracture healing are somewhat analogous to wound healing in other tissues. Following an inflammatory phase, there is a mesenchymal and angiogenic activation phase. Blood vessels and mesenchymal stem cells (MSCs) are recruited to the injury site and proliferate. This is followed by a phase that is unique to bone regeneration. MSCs differentiate to become either chondrocytes or osteoblasts (Fig. 1). Osteoblasts directly deposit bone via a process termed intramembranous ossification, while chondrocytes proliferate, hypertrophy, mineralize, and then new bone is deposited onto the cartilaginous matrix through a process termed, endochondral ossification. The process is completed by remodeling of the bone to restore normal shape and function. MSCs are a critical component of the bone-healing 
process as they represent the precursors for both osteoblasts and chondrocytes, and likely also modulate the healing response. This review will examine the role of MSC in fracture healing and will discuss how MSC may be manipulated and utilized to improve bone healing.

\section{TRANSLATIONAL RELEVANCE}

The use of MSCs to heal bone is being actively investigated in the treatment of bone disorders and injuries. These studies require the utilization of comparative animal models. MSCs have been studied in a wide variety of animal species, including, but not limited to, mice, rats, rabbits, dogs, pigs, and horses. As in humans, MSCs can be isolated in these animal models and thus these animals have been valuable translational models to investigate the clinical usefulness of MSCs for bone regeneration. Translational studies have been utilized to investigate approaches for sites of isolation, culture approaches, and mechanisms of delivery. The review of these studies forms the basis for much of this review.

\section{CLINICAL RELEVANCE}

MSCs, whether isolated and delivered for a therapeutic effect, or activated using biological or pharmacological means, show great potential clinical promise. To date, the therapeutic delivery of MSCs to repair bone has not received approval from the U.S. Food and Drug Administration, although there have been isolated reports from clinical groups outside of the United States that describe the delivery of MSCs to repair bone. However, products that utilize "non-manipulated" cell preparations that likely contain MSCs are currently commercially available. Similarly, other therapeutic approaches that seek to activate the growth and differentiation of MSCs in vivo using various growth factors are currently on the market or are in clinical trials.

\section{DISCUSSION OF FINDINGS AND RELEVANT LITERATURE Mesenchymal stem cells}

Bone marrow is most commonly associated with its abundant hematopoietic precursors, but it is also where MSCs were originally demonstrated. The first evidence of MSCs was the report by German pathologist Julius Friedrich Cohnheim in 1867 on "mesenchymal precursor cells" that were fibroblastoid, adherent, and extravasated at sites of tissue injury. ${ }^{2}$ Goujon found that bone marrow from rabbits and chickens could create sites of ossification when transplanted into muscle. ${ }^{3}$ A century later these ossifying cells that Cohnheim and Goujon had observed were finally isolated in cell culture and described by their plasticadherent, colony-forming cell populations harvested from the bone marrow compartment. ${ }^{4}$ In the early $1990 \mathrm{~s}$, the cells began to be referred to as "mesenchymal stem cells" when they were shown to exhibit multipotential differentiation capacity-possessing osteoblastic adipocytogenic and chondogenic potential, in addition to self-renewal. ${ }^{5}$ These findings brought MSCs into the spotlight-both as a potential orthopedic therapy, via manipulation of MSC osteogenic capabilities endogenously, and in direct application of MSCs to increase osteogenesis in bone repair.

MSCs have now been isolated from many sites throughout the body. In the bone compartment, they can be found in the bone marrow, periosteum, and endosteum, thin connective tissue linings of the surface of bones, and the mineralized bone itself, and are known to be the primary sources of cells during bone repair (Fig. 2). There are also many additional sources of

\author{
Abbreviations \\ and Acronyms (continued) \\ IGF-1 = insulin-like growth \\ factor 1 \\ $\mathrm{LRP}=$ low-density lipoprotein \\ receptor-related protein \\ $\mathrm{MSC}=$ mesenchymal stem cell \\ $\mathrm{NICD}=$ notch intracellular \\ domain \\ $\mathrm{P}-\mathrm{MSC}=$ periosteal-derived \\ mesenchymal stem cells \\ $\mathrm{PRP}=$ platelet-rich plasma \\ TGF- $\beta=$ transforming growth \\ factor beta
}




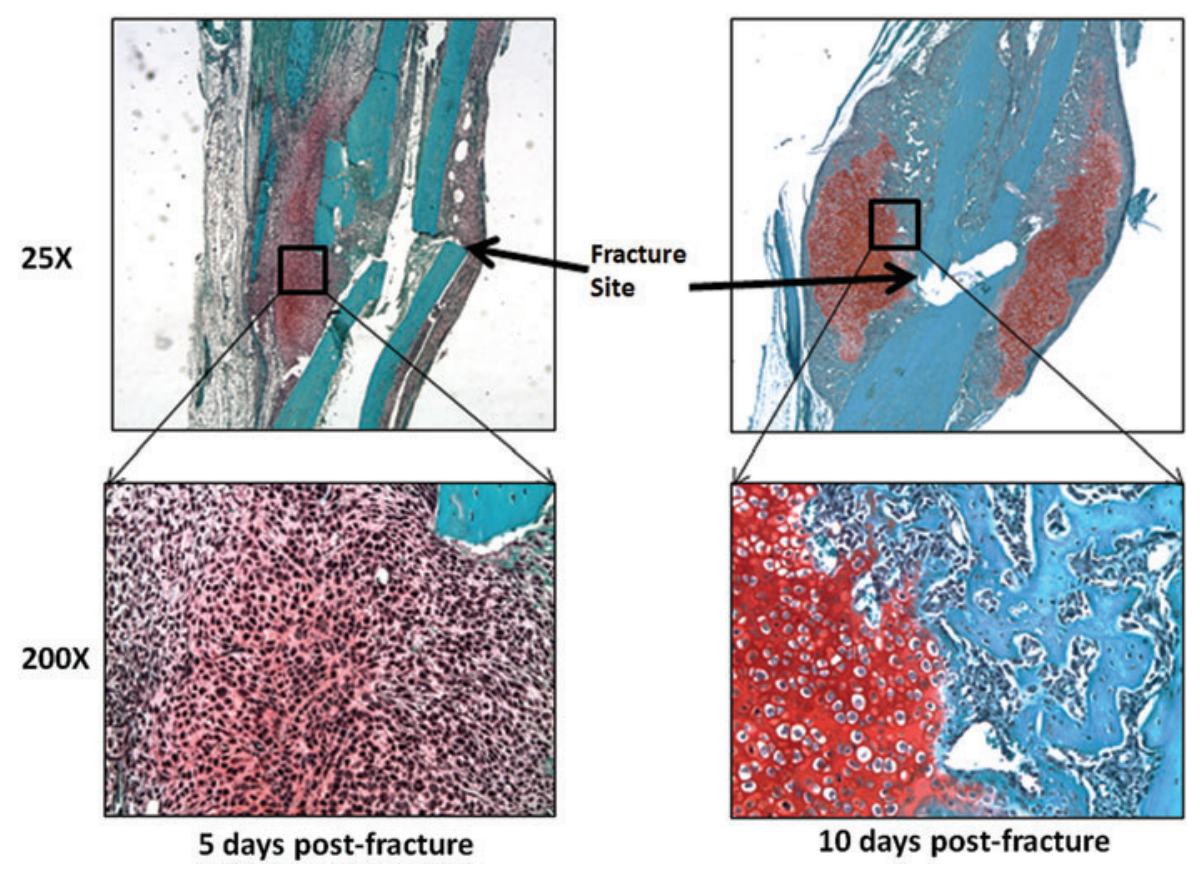

Figure 1. Mesenchymal phase of fracture regeneration. Bones were harvested from mice 5 and 10 days postfracture. Callus tissue was decalcified, embedded in paraffin, sectioned, and stained with saffranin-0. Images were collected at $25 \times$ and $200 \times$ magnification to demonstrate the mesenchymal phase at day 5 , and then how those cells differentiate to become bone-forming cells at day 10 . To see this illustration in color, the reader is referred to the web version of this article at www.liebertpub.com/wound

MSCs in the body that likely do not contribute to endogenous bone repair such as adipose tissue, muscle, and synovium. The comparative quality and character, as well as the differentiation potential of MSCs from each of these sources varies and is still debated. ${ }^{6}$

MSCs derived from bone marrow. The first identified source of a cell referred to as an MSC was the bone marrow (BM-MSC), and bone marrow remains a common source of cells. BM-MSCs are rare compared to other cell types in the bone marrow, so one must isolate and separate them from the plentiful hematopoietic cells in the collected aspirates. In vitro culture of these cells from rats reveals that they form fewer colonies than MSCs from adipose or periosteum (A-MSC and P-MSC, respectively), but like $\mathrm{P}-\mathrm{MSCs}$, they show more mineralization and a higher osteocalcin content-a marker of late osteoblast differentiation. BMMSCs also have the highest alkaline phosphatase (ALP) levels at early time points, which is a marker of early osteoblast differentiation. ${ }^{7}$ Canine BMMSCs show less MSC yield per gram of tissue as compared to canine P-MSCs, ${ }^{8}$ while human BMMSCs have fewer colonies like the rat cells, but proliferate rapidly to develop into populations of similar number to MSCs from other tissues due to their great expansion properties. ${ }^{9}$ Bone marrow can be readily accessed in comparatively plentiful quantities for use in expansion and therapeutics, which is one of the reasons that its use is very common in orthopedic surgery. ${ }^{6}$ Particularly, it is likely that orthopedic surgeons are comfortable with the concept of using marrow-derived MSCs because cancellous bone is frequently used as autograft material in bone regeneration.

MSCs derived from mineralized bone. MSCs can also be directly isolated from mineralized bone. The first studies utilized trabecular bone from within the marrow cavity to isolate MSCs. Bone is harvested either via curettage or reaming of the marrow cavity. Collected bone chips are washed of marrow contamination and digested with collagenase in MSC media. The media is filtered to remove the bone chips, and the cells that remain are permitted to proliferate. ${ }^{10}$ As an alternative to this, the bone chips can be plated directly. Cells derived from bone are able to differentiate into osteoblasts, chondrocytes, and adipocytes, and have similar gene expression levels as BM-MSCs both before and after differentiation. ${ }^{11}$ Trabecular bone samples contain similar numbers of nucleated cells relative to bone marrow aspirates of the same size, but the trabecular bone samples are able to form significantly more colonies, suggesting the presence of more MSCs. ${ }^{10}$ 


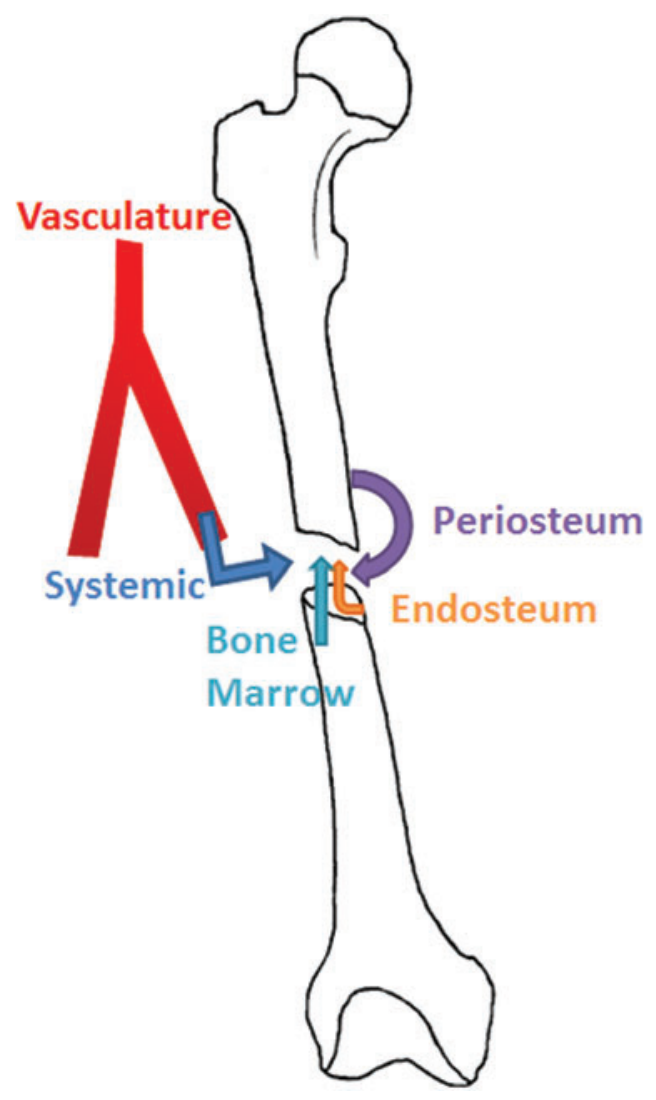

Figure 2. Endogenous sources of mesenchymal stem cells (MSCs) that contribute to fracture repair. MSCs that contribute to the fracture site are derived from the periosteum, endosteum, and the bone marrow. As well, MSCs present in the systemic circulation may also contribute to healing. To see this illustration in color, the reader is referred to the web version of this article at www.liebertpub.com/wound

Recently, compact bone has become a source for MSCs (B-MSCs), with many researchers supporting the advantages of using these rather than BMMSCs. To culture them, the bone is washed of any marrow contamination, cut into small fragments, and digested with collagenase. The bone chips are washed of the collagenase-digested cells and cultured in a plastic dish with MSC media. The B-MSCs grow out of the bone chips and adhere to the dish surrounding the chips. These cells form colonies as early as 3 days after plating, whereas the BM-MSCs are only seen as single cells at this point. Cultured B-MSCs also have less hematopoietic cell contamination and require fewer media changes. B-MSCs are similar to BM-MSCs in differentiation capabilities and cell surface markers. ${ }^{12}$ Taken together, B-MSCs can be expanded quickly and produce a more pure yield, both of which could be advantageous for therapeutic implantation.

MSCs derived from periosteum. The periosteum was identified as a source of MSCs very early, but only recently has gained interest as a potentially better source for MSCs than from marrow or bone. ${ }^{13}$ It is recognized as one of the most crucial components of bone healing by orthopedic surgeons, who take care to not disturb or remove it around sites of injury. ${ }^{14}$ Even from the earliest reports, these cells were shown to be able to differentiate into bone and cartilage. ${ }^{13}$ In a recent canine study, P-MSCs had the highest cell mass produced per unit of harvested tissue when compared to BM-MSCs and A-MSCs. ${ }^{8}$ The periosteum coats all bone surfaces except for locations of articular cartilage, muscle and tendon insertions, and sesamoid bones. It is therefore plentiful, but can be difficult to harvest due to the cambrium layer being strongly attached to the bone surface. It is generally considered a more difficult tissue to harvest compared to bone marrow, ${ }^{6}$ but can be readily accessed in certain surgical procedures. Due to its large in vivo contribution to production of new osteoblasts and chondrocytes locally, this tissue has a lot of promise as a source of MSCs for therapeutics. ${ }^{15}$

MSCs derived from adipose. Adipose, although likely not an endogenous source of MSCs for in vivo bone repair, presents another possible source for therapeutic use. Similar to the other sources, MSCs from adipose (A-MSCs) are able to undergo osteogenic, chondrogenic, and adipogenic differentiation. Also similar to other sources, their relative ability to undergo differentiation is variable based on the isolation method and species. Various studies have suggested that the endogenous osteogenic potential of A-MSCs is less compared with BM-MSCs. As one example, in rats, A-MSCs have a significantly lower ALP activity per DNA than BM-MSCs and P-MSCs, and osteocalcin levels follow the same trend. ${ }^{16}$ When A-MSCs were implanted in muscle, ectopic bone formed at the site, but it was less than that formed with BM-MSCs or P-MSCs. This group concluded that A-MSCs show low endogenous osteoblast differentiation in vivo, but instead recruit other cells that increased bone formation. This suggests that A-MSCs may play a role in recruiting other cell types to form bone. In a related manner, genetically transduced A-MSCs may be useful for delivery of bone inducing growth factors, such as BMP. For example A-MSCs with forced expression of BMP-2 show increased bone regeneration when transplated in vivo, ${ }^{17}$ but it is most likely that the mechanism of action was through recruitment of host cells, because delivery of BMP-2 as a growth factor or gene in similar regeneration models can also induce bone formation. Despite likely limited endogenous osteoblast differentiation potential, one 
of the great qualities of A-MSCs is that their source is plentiful. Adipose is easily harvested through lipoaspiration and can be used to isolate A-MSCs. ${ }^{18}$

\section{MSCs during bone regeneration}

Bone regeneration is a complex process involving cells and factors from many different compartments of the bone. The current research supports that both local and distant progenitor cells contribute to repair, and a variety of soluble factors are critical components of bone repair. As discussed previously, only some sources of stem cells contribute to bone regeneration in vivo (Fig. 2). Although MSCs from fat and muscle can differentiate into osteoblasts and chondrocytes in vitro, they have not been demonstrated as contributing to bone repair in vivo, ${ }^{6}$ although some controversy exists as to whether muscle may also contribute MSCs to repair.

Bone marrow contribution to healing. Bone marrow is known to contribute to bone repair after an injury as its removal delays fracture repair, but the mechanism by which this occurs is debated. ${ }^{19}$ Taguchi et al. created a green fluorescent protein (GFP)-chimeric mouse, having received a bone marrow transplant (BMT) from a GFP transgenic mouse. After transverse femoral fracture, GFPpositive cells were incorporated into the fracture callus (but not in the uninjured bone), and were more plentiful centrally in the callus where the osteocalcin-positive osteoblasts were located. These data suggest that bone marrow is a source of MSCs, which serve as progenitors to osteoblasts in the callus, particularly near to the fracture ${ }^{20}$ (Fig. 2). However, in a similar experiment, marrow was transplanted from a Rosa26 mouse (constitutively expressing LacZ) into a wild-type mouse, and tibiae were fractured. Chondrocytes and osteoblasts in the calluses were not found to be X-gal-positive, but a large number of inflammatory and osteoclast lineage cells were present and X-gal-positive. This suggests that bone marrow may have a role in fracture repair other than providing MSCs, potentially acting to aid in differentiation of MSC derived from another source. ${ }^{21}$ Finally, in a more recent study, lineage analysis has clearly showed that bone marrow MSCs directly contribute to becoming osteoblasts and osteocytes ${ }^{15}$ (Fig. 3). During the previously discussed BMT experiment, stromal cells in the bone marrow may not have been irradiated; thus, donor MSCs may not have had a niche to occupy in the host. Additionally, the ephemeral nature of transplanted MSCs in bone marrow has been noted previously-either of these explanations could have resulted in these discordant findings. ${ }^{22}$

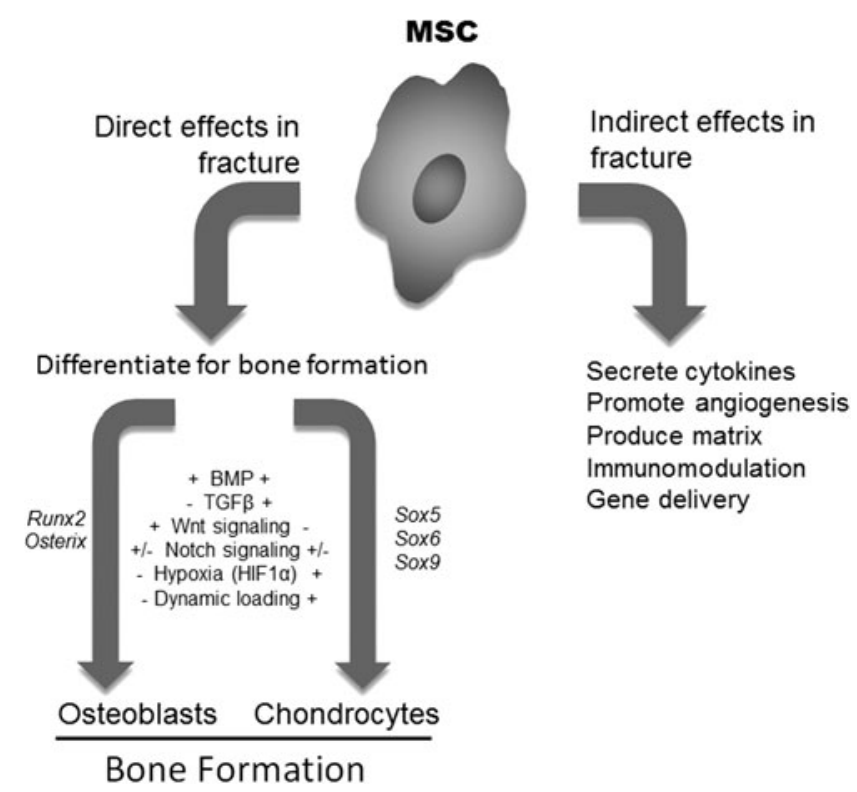

Figure 3. MSCs in fracture directly form osteoblasts and chondrocytes, but also may have indirect effects. MSCs are the cells that develop to become osteoblasts and chondrocytes. A variety of hypothesized and documented factors contribute to the differentiation process. These factors result in the modulation of transcription factors that are specific for the osteoblast and chondrocyte differentiation. MSCs at the fracture site may also contribute to bone healing through indirect effects, by producing cytokines, growth factors and regulating vascularization and modulating inflammation.

Periosteal contribution of MSCs to healing. The periosteum is also known to be a major contributor to bone repair (Fig. 2). Removal of the periosteum from the bone surface delays healing. ${ }^{19,23}$ After injury, periosteal cells proliferate extensively and are known to contribute to both the formation of cartilage and bone in the fracture callus (Fig. 3). Removal of the periosteum causes the time to cartilage formation at the fracture site to double. ${ }^{19}$ Another group confirmed that osteoblasts involved in fracture repair come from the periosteum by tracing their lineage via alpha smooth muscle actin $(\alpha \mathrm{SMA})-C r e$ expression. This group of investigators has demonstrated that $\alpha$ SMA-Cre, while not specific for MSCs, is a useful marker for precursors that will develop into both chondrocytes and adipocytes. These cells are associated with the microvasculature as well as dispersed in the periosteum in a fibroblastoid conformation. ${ }^{24}$ Similarly, disruption of the periosteum, but not the bone marrow or endosteum, inhibited cartilage production and endochondral ossification, further supporting a local source of cells coming from the periosteum. The periosteum is the primary source of chondrocytes in the callus in a gap defect model, and this remains true even if a graft is reversed so that the 
periosteum has an endosteal location. This confirms that cells from the periosteum possess a unique chondrogenic potential and that under normal healing conditions, bone marrow is not inhibiting or the soft tissue stimulating this ability. Rather it is a cell-autonomous function of P-MSCs. ${ }^{15}$

Circulatory cell contribution to healing. Circulating cells are also theorized to contribute to bone repair (Fig. 2). Bone morphogenetic proteins (BMPs) are known to be an essential component in recruiting cells to areas of bone repair, and BMP2collagen pellets placed in muscle induce MSC migration to the pellet from the bone marrow. Additionally, these circulating cells differentiate and become osteoblasts within the ectopic bone. ${ }^{25}$ Definitive proof that endogenous MSCs could migrate from the bone marrow to the systemic circulation was provided by Pitchford et al. with the injection of a CXCR4 antagonist. ${ }^{26}$ In another study, BM-MSC in systemic circulation are able to home to the site of an injury, but do not differentiate into osteoblasts or chondrocytes and contribute to the callus as one might expect given their behavior in vitro. ${ }^{27}$

\section{Signals regulating MSCs during bone regeneration}

The signaling for the migration, proliferation, and differentiation of MSCs to the site of injury is complex and has not yet been fully elucidated. However, many factors are known to influence these processes, including fibroblast growth factor, BMP, Wnt/ $\beta$-catenin, and Notch signaling pathways, as well as other physiologic stimuli, such as hypoxia and mechanical loading (Fig. 3). By harnessing growth factors, either in combination with exogenous MSCs or alone to stimulate endogenous MSCs, therapeutics could be developed to promote MSC proliferation and differentiation to heal fractures and treat conditions of low bone mass.

BMPs. BMP family members stimulate MSC differentiation and proliferation. BMPs belong to the transforming growth factor beta (TGF- $\beta$ ) superfamily, and there are currently 13 identified BMPs. BMPs bind two different BMP receptor classes that exist as heterodimers, which leads to phosphorylation of Smad proteins. Phosphorylation activates the Smad proteins, enabling their activity as transcription factors for many target genes. ${ }^{28}$ One of the characteristic target genes is Runx2, one of the master regulators of osteogenesis. BMP2, BMP4, BMP6, BMP7, BMP9, BMP12, and BMP13 have been shown to induce differentiation of MSCs, and BMP3 induces MSC prolifera- tion. ${ }^{29}$ Deletion of BMPs both individually and together in mice leads to skeletogenesis defects, and osteogenesis ceased when BMP2 and BMP4 are disrupted together. ${ }^{30}$ Specific deletion of BMP2 in the limbs allows for normal skeletal patterning and cartilage-to-bone transition prenatally, but postnatally there is a delay in the development of the secondary ossification center and defects in collagen at the articular surface. Upon weightbearing, the mice develop spontaneous limb fractures, and upon experimental fracture, they completely fail to form a bridging callus. ${ }^{31} \mathrm{~A}$ similar experiment was also completed with BMP4, and its disruption in limb cells did not result in a problem with fracture healing. ${ }^{32}$ This suggests that the role of endogenous BMPs in fracture healing is variable, and may be dependent upon the cell type producing the specific BMP.

Wnt signaling. Wnt/ $\beta$-catenin signaling regulates development, cell proliferation, cell fate determination, and cell polarity across all organisms examined. In canonical Wnt signaling, Wnt molecules signal through Frizzled and Low-density lipoprotein receptor-related protein (LRP) coreceptors, leading downstream to the escape of $\beta$-catenin from degradation. $\beta$-catenin is then able to accumulate in the nucleus and interact with the T-cell-specific factor and the lymphoid enhancerbinding factor 1 to alter gene transcription. In contrast, noncanonical Wnt signaling does not operate through $\beta$-catenin. ${ }^{33}$ In bone, the role of canonical Wnt signaling is debated and has both been seen to promote proliferation and differentiation, as well as to inhibit proliferation via Wnt3a. ${ }^{34,35}$ One study suggests that this effect may be concentration-dependent, with low doses of Wnt stimulating proliferation and high doses of Wnt inhibiting it. $^{36}$ The noncanonical Wnt5a suppresses $\beta$-catenin signaling and causes inhibition of MSC proliferation. ${ }^{37}$ While it is clear that Wnt signaling is an important regulator of MSC proliferation, the mechanism appears to be complex and is not yet fully elucidated. The story regarding Wnt signaling and osteogenesis appears to be similarly complex. Noncanonical Wnt11 appears to play a role in osteogenic differentiation and is upregulated during this process in vitro, while the canonical Wnt3a suppresses MSC osteogenic differentiation and reduces matrix deposition and ALP activity. ${ }^{34}$ However, another study found that while low levels of Wnt3a did suppress osteogenic differentiation, high levels were able to stimulate it. $^{36}$ Additionally, Wnt6, Wnt10a, and Wnt10b are reported to stimulate osteoblastogenesis in a 
canonical, $\beta$-catenin-dependent manner. ${ }^{38}$ The effects of Wnt signaling on MSC proliferation and osteogenic differentiation are complex and likely sensitive to specific Wnt ligand, culture conditions, species, concentrations, and other factors present, leading to the current contradictory reportings.

Notch signaling. Notch signaling regulates development in mammals through cell differentiation, proliferation, and apoptosis. Notch is a transmembrane receptor that interacts with membrane-bound Delta and Jagged on neighboring cells. This causes the proteolytic cleavage of the Notch intracellular domain (NICD) by $\Upsilon$-secretase, and NICD then translocates to the nucleus to complex with CSL. CSL, along with coactivator Mastermind-like (MAML), activates transcription of target genes, including genes of the Hey and Hes families. ${ }^{39}$ Overexpression of NICD in mice leads to an osteosclerotic phenotype and an increase in the number of osteoblast precursors, while Notch signaling knockout mice have an osteoporotic phenotype and increased numbers of osteoclasts. ${ }^{40}$ Additionally, Notch signaling shows crosstalk with other osteogenic pathways, which leads to further involvement in regulation of osteogenesis. ${ }^{41}$ The role of Notch signaling during bone regeneration has not been reported, but preliminary results suggest that inhibition of Notch signaling results in smaller fracture calluses.*

\section{Manipulating MSCs to heal bone}

Skeletal injuries are a source of significant debilitation, particularly with hard-to-heal fractures, such as those occurring in the aging population, and those with associated vascular injury. Autologous bone grafts (ABGs) are often employed to help in these circumstances, and have been shown to improve repair via fracture stabilization by providing a scaffold for new bone growth (osteoconduction), MSCs and osteoblasts (osteogenesis), and some growth factors to stimulate new bone growth (osteoinduction). However, increasing the endogenous osteopotential and eliminating the need for ABG harvest, which is associated with donor-site morbidity, would be highly advantageous. ${ }^{42}$ Utilizing both MSC cellular therapies and growth factors to manipulate the endogenous cell behavior in vivo has incredible potential for use in the clinic.

\footnotetext{
*Dishowitz MI: Inhibition of notch signaling during bone regeneration, University of Pennsylvania, Philadelphia, PA, 2013 (personal communication).
}

Growth factors to activate MSCs in fracture healing. The use of growth factors to activate MSCs at the fracture site can increase osteoinduction and lead to a faster recovery (Fig. 4). A common mixed source of osteoinductive agents used in fracture repair is platelet-rich plasma (PRP), although the efficacy of this treatment is controversial. PRP contains many growth factors, including TGF $\beta 1$, TGF $\beta 2$, platelet-derived growth factor, insulin-like growth factors (IGFs), and epidermal growth factor, and has been seen to improve fracture repair to a similar extent as treatment with MSCs alone. ${ }^{43}$ The amount of growth factor present is smaller compared to ABG and contains little of the most potent osteoinducer, BMP. Additionally, there can be large variations in concentrations of these factors from one sample to the next. ${ }^{44}$

Demineralized bone matrix (DBM) is another product commonly utilized for fracture repair that contains many osteoinductive growth factors, but no cellular component. The collagen matrix of DBM provides some osteoconductive properties, and again, only small amounts of the most osteoinductive growth factor, BMP, are present with great variability from one product to another. ${ }^{45}$

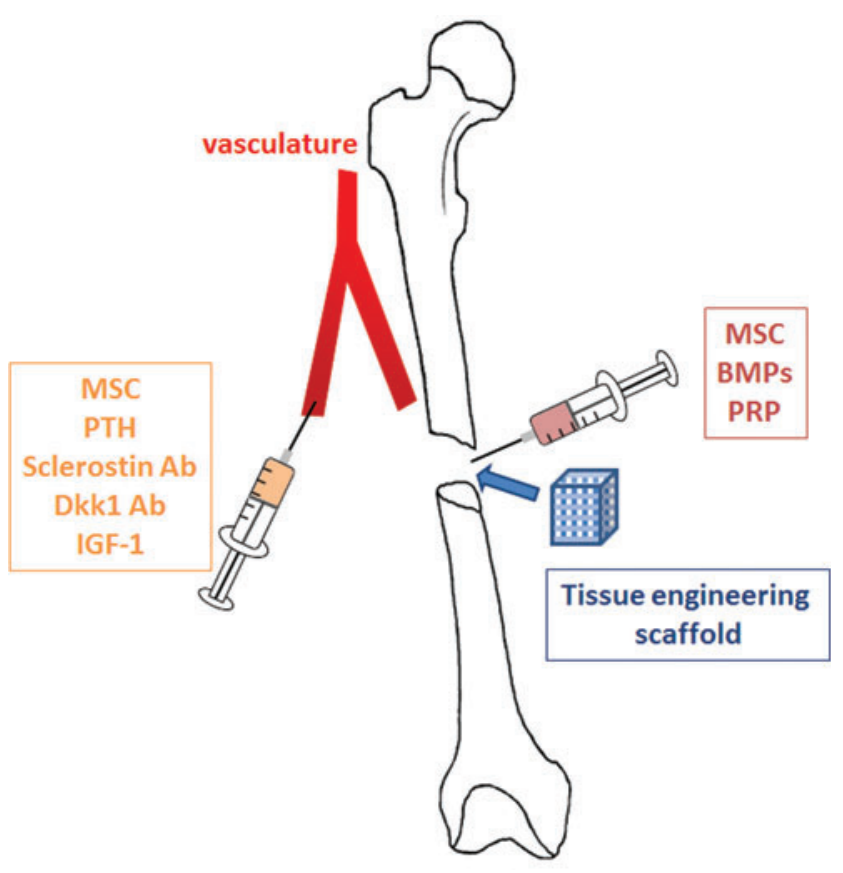

Figure 4. Exogenous osteogenic, osteoinductive, and osteoconductive factors manipulating MSCs that could be used to promote fracture repair. Factors or MSCs could be delivered systemically through the circulation, or alternatively MSCs or factors can be directly delivered to a fracture site. Local delivery may involve direct injection or the loading of factors in scaffolds. To see this illustration in color, the reader is referred to the web version of this article at www.liebertpub.com/wound 
BMPs are now commercially available and are commonly used for nonunions for their osteoinductive properties. BMP therapies in many instances are combined with an osteoconductive scaffold. Although many of the BMPs are known to be osteoinductive as noted above, only BMP2 and BMP7 are currently U.S. Food and Drug Administration-approved for use in bone repair, with delivery on collagen sponges. ${ }^{44}$ This method requires the fracture site to be open, but other techniques such as injectable pastes and gene therapy approaches are being tested in the laboratory, and these could eliminate the need for an open site. ${ }^{17,46}$ Osteoinductive coatings for fixation devices are also being developed and would offer the benefit of combined therapy (fixation and factor delivery) as well as a more sustained delivery. ${ }^{47}$

Due to their potent osteoinductive effects even at ectopic sites, BMPs cannot be given systemically. However, the Wnt signaling pathway can be stimulated systemically to increase bone (Fig. 4). The parathyroid hormone is known to increase bone on all surfaces through the Wnt pathway, and has been used both in the clinic to increase bone in osteoporotic patients, as well as in the laboratory to increase fracture strength. ${ }^{48,49}$ Another potential therapeutic is an antibody to the secreted Wnt protein Dickkopf 1 (Dkk1). Dkk1 binds to the LRP receptors and inhibits signaling, so relieving this inhibition can lead to increased bone as seen in a murine fracture model with increased callus size, biomechanical properties, and bone mineral density when Dkk1 was administered subcutaneously at the time of fracture. ${ }^{50}$ Sclerostin works in a similar manner to Dkk1 in Wnt signaling, and also shows promise in increasing bone formation. ${ }^{51}$ IGF1 has also been administered subcutaneously in mice and was found to increase fracture callus size and biomechanical properties. ${ }^{52}$ There are other potential options for enhancement of fracture healing via either local or systemic treatments, but many of these still need to be tested for side effects outside of bone.

Delivery of MSCs to promote healing. Direct delivery of harvested MSCs is another strategy for improving bone repair (Fig. 4). ABGs and bone marrow aspirates are some of the easiest ways to deliver MSCs to fracture sites, although the number of cells present is limited in the tissues obtained. The time to union in a nonunion fracture was inversely related to the concentration of MSCs, and the size of the callus was directly related to the number of MSCs injected in one study. For optimal repair, the concentration of the source of MSCs through smaller aspirate collection and centrifugation was needed to achieve satisfactory unions. ${ }^{53}$ Bone marrow aspirates contain many osteoinductive growth factors as well as osteoconductive MSCs, but are lacking in an osteoconductive scaffold.

Another option is to use isolated and cultivated MSCs directly (Fig. 4). The many sources and variability in the characteristics of MSCs were previously discussed. While MSCs are osteogenic, they do require an osteoconductive environment to be able to proliferate and differentiate. Due to this, they are often utilized in combination with an osteoconductive scaffold (tissue engineering strategy). Using MSCs alone rather than a harvested tissue like bone marrow allows for isolation, culture, and greater expansion of the cells, and thus, a higher number and concentration delivered, which increases the osteogenic effects. ${ }^{53}$

The benefits of delivering MSCs on a scaffold have been validated in many animal models, including in a canine model with a ceramic scaffold. This model increased the ability of a large defect to heal in union rather than nonunion, and demonstrated bone both filling the pores of the implant as well as forming a bony collar around it that was incorporated into the callus. ${ }^{54}$ Since then, many different scaffolds have been tested and the induction of differentiation of MSCs before implantation has been tested. Indeed, osteoblast preinduction will result in more of the implanted cells becoming osteoblasts. Alternatively, inducing chondrogenesis via TGF $\beta$ allowed for endochondral ossification, rather than the intramembranous ossification seen in other studies with either little or no osteoblast induction of the implanted $\mathrm{MSCs}^{55}$

Exogenous MSCs can also be delivered through percutaneous injection directly into a bone-healing site, or administered in the systemic circulation (Fig. 4). MSCs will home directly to sites of bone injury when administered systemically, but they have not been shown to directly contribute to healing in this context by becoming bone-forming cells. ${ }^{56}$ However, delivered MSCs may have indirect effects on healing. While not a substantial focus of this review, MSCs, whether endogenous or exogenous, can likely play a modulatory role in fracture healing by recruiting other cell-types and increasing vascularization and by modulating immunological responses ${ }^{57}$ (Fig. 3). The use of MSCs directly for bone regeneration clearly has great potential, but more studies need to be done to demonstrate a beneficial effect; safety, the proper site of harvest and purification methodologies, delivery techniques, and more studies need to be 
done to examine consistency in the MSC populations isolated and used for therapeutics.

\section{SUMMARY}

Harnessing MSCs to improve fracture healing has a profound potential clinic benefit. Full understanding of the pathways that govern MSC proliferation and differentiation is still being clarified, and will be necessary for the full development of therapeutics to manipulate MSCs in vivo, as well as ex vivo for reimplantation. Standardization of MSCs from the various sources is also necessary to better characterize the concentration and number of cells needed, as well as to fully clarify the effects of these techniques without confounding variables. Utilization of MSCs and their signaling factors has potential for a significant impact on the treatment of hard-to-heal fractures in the clinic and is a very exciting segment of wound care research. However, development of robust preclinical models for assessing ideal therapeutic approaches to manipulate MSCs is an absolute necessity. While murine models are useful for assessing mechanistic aspects of bone healing, utilization of larger animal models will be absolutely essential for defining ideal therapeutic approaches.

\section{ACKNOWLEDGMENTS AND FUNDING SOURCES}

The authors would like to thank Lucas Lopas (University of Wisconsin, Madison, WI) for assistance with figures, and Jonathan Madara (University of Pennsylvania) for critical review of the manuscript. The authors would like to apologize to our many colleagues whose excellent work could not be cited due to formatting constraints.

\section{AUTHOR DISCLOSURE AND GHOSTWRITING}

No competing financial interests exist for the authors. The content of this article was expressly written by the authors listed. No ghostwriters were used to write this article.

\section{ABOUT THE AUTHORS}

M. Noelle Knight is a student in the Veterinary Medical Scientist Training Program at the University of Pennsylvania. She is currently working toward both her VMD and $\mathrm{PhD}$ degrees through the School of Veterinary Medicine and the Perelman School of Medicine. She graduated with honors from Dartmouth College in 2008, receiving her Bachelor's Degree in Cellular Biology. Noelle has completed 3 years of her veterinary education and has an interest in equine orthopedics. She is currently working on her predoctoral research in Dr. Hankenson's laboratory. Her research is focused on promoting bone formation through elucidation and manipulation of the Wnt signaling pathway in mesenchymal stem cells. Specifically, she is interested in the role of $\mathrm{R}$-spondin 2 in bone maintenance and repair. Dr. Kurt D. Hankenson is the Dean W. Richardson Chair for Equine Disease Research at the University of Pennsylvania. He received his DVM from the University of Illinois, and then following equine practice, he received a $\mathrm{PhD}$ in the biochemistry of the extracellular matrix at the University of Washington working with Dr. Paul Bornstein. Dr. Hankenson has been an independent scientist since 2002, first at the University of Michigan, and currently at the University of Pennsylvania. Dr. Hankenson has extensive experience working with genetargeted and transgenic murine models and in the cellular and molecular regulation of osteoblastogenesis. Dr. Hankenson's laboratory studies the regulation of mesenchymal progenitor differentiation and maintenance of progenitor cell stemness, focused primarily on signaling factors regulating osteoblast differentiation (growth factors). His work has characterized the regulation of osteoblast differentiation by BMP, Wnt, and Notch signaling. His work utilizes various rodent models of bone formation, including several regeneration models. 


\section{REFERENCES}

1. Marzona $L$ and Pavolini B: Play and players in bone fracture healing match. Clin Cases Miner Bone Metab 2009; 6: 159.

2. Cohnheim J: Über entzündung und eiterung. Path Anat Physiol Klin Med 1867; 40: 1.

3. Goujon E: Recherches experimentales sue les proprieties. J L Anat 1869; 6: 399.

4. Friedenstein AJ, Chailakhjan RK, and Lalykina KS: The development of fibroblast colonies in monolayer cultures of guinea-pig bone marrow and spleen cells. Cell Tissue Kinet 1970; 3: 393.

5. Caplan Al: Mesenchymal stem cells. J Orthop Res 1991; 9: 641.

6. Colnot $C$ : Cell sources for bone tissue engineering: insights from basic science. Tissue Eng Part $B$ Rev 2011; 17: 449.

7. Ito T, Sawada R, Fujiwara Y, and Tsuchiya T: FGF2 increases osteogenic and chondrogenic differentiation potentials of human mesenchymal stem cells by inactivation of TGF-beta signaling. Cytotechnology 2008; 56: 1.

8. Kisiel AH, McDuffee LA, Masaoud E, Bailey TR, Esparza Gonzalez BP, and Nino-Fong R: Isolation, characterization, and in vitro proliferation of canine mesenchymal stem cells derived from bone marrow, adipose tissue, muscle, and periosteum. Am J Vet Res 2012; 73: 1305

9. Sakaguchi Y, Sekiya I, Yagishita K, and Muneta T: Comparison of human stem cells derived from various mesenchymal tissues: superiority of synovium as a cell source. Arthritis Rheum 2005; 52 : 2521.

10. Sakaguchi Y, Sekiya I, Yagishita K, Ichinose S, Shinomiya K, and Muneta T: Suspended cells from trabecular bone by collagenase digestion become virtually identical to mesenchymal stem cells obtained from marrow aspirates. Blood 2004; 104: 2728.

11. Tuli R, Seghatoleslami MR, Tuli S, Wang ML, Hozack WJ, Manner PA, Danielson KG, and Tuan RS: A simple, high-yield method for obtaining multipotential mesenchymal progenitor cells from trabecular bone. Mol Biotechnol 2003; 23: 37.

12. Zhu H, Guo ZK, Jiang XX, Li H, Wang XY, Yao HY, Zhang $Y$, Mao N: A protocol for isolation and culture of mesenchymal stem cells from mouse compact bone. Nat Protoc 2010; 5: 550.

13. Nakahara H, Dennis JE, Bruder SP, Haynesworth $\mathrm{SE}$, Lennon DP, and Caplan Al: In vitro differentiation of bone and hypertrophic cartilage from periosteal-derived cells. Exp Cell Res 1991; 195: 492.

14. Gerstenfeld LC, Cullinane DM, Barnes GL, Graves DT, and Einhorn TA: Fracture healing as a postnatal developmental process: molecular, spatial, and temporal aspects of its regulation. J Cell Biochem 2003; 88: 873.
15. Colnot C: Skeletal cell fate decisions within periosteum and bone marrow during bone regeneration. J Bone Miner Res 2009; 24: 274.

16. Hayashi $O$, Katsube $Y$, Hirose $M$, Ohgushi $H$, and Ito $\mathrm{H}$ : Comparison of osteogenic ability of rat mesenchymal stem cells from bone marrow, periosteum, and adipose tissue. Calcif Tissue Int 2008; 82: 238.

17. Li H, Dai K, Tang T, Zhang X, Yan M, and Lou $\mathrm{J}$ : Bone regeneration by implantation of adipose-derived stromal cells expressing BMP2. Biochem Biophys Res Commun 2007; 356: 836.

18. Levi B and Longaker MT: Concise review: adiposederived stromal cells for skeletal regenerative medicine. Stem Cells 2011; 29: 576.

19. Ozaki A, Tsunoda M, Kinoshita S, and Saura R: Role of fracture hematoma and periosteum during fracture healing in rats: interaction of fracture hematoma and the periosteum in the initial step of the healing process. J Orthop Sci 2000; 5: 64.

20. Taguchi K, Ogawa R, Migita M, Hanawa $H$, Ito $H$, and Orimo $\mathrm{H}$ : The role of bone marrow-derived cells in bone fracture repair in a green fluorescent protein chimeric mouse model. Biochem Biophys Res Commun 2005; 331: 31.

21. Colnot C, Huang S, and Helms J: Analyzing the cellular contribution of bone marrow to fracture healing using bone marrow transplantation in mice. Biochem Biophys Res Commun 2006; 350: 557.

22. Dominici M, Marino R, Rasini V, Spano C, Paolucci P, Conte P, Hofmann TJ, and Horwitz EM: Donor cell-derived osteopoiesis originates from a selfrenewing stem cell with a limited regenerative contribution after transplantation. Blood 2008; 111: 4386.

23. Utvåg SE, Grundnes 0, and Reikeraos 0: Effects of periosteal stripping on healing of segmental fractures in rats. J Orthop Trauma 1996; 10: 279 .

24. Grcevic D, Pejda S, Matthews BG, Repic D, Wang L, Li H, Kronenberg MS, Jiang X, Maye P, Adams DJ, Rowe DW, Aguila HL, and Kalajzic I: In vivo fate mapping identifies mesenchymal progenitor cells. Stem Cells 2012; 30: 187.

25. Otsuru S, Tamai K, Yamazaki T, Yoshikawa H, and Kaneda Y: Bone marrow-derived osteoblast progenitor cells in circulating blood contribute to ectopic bone formation in mice. Biochem Biophys Res Commun 2007; 354: 453.

26. Pitchford SC, Furze RC, Jones CP, Wengner AM, and Rankin SM: Differential mobilization of subsets of progenitor cells from the bone marrow. Cell Stem Cell 2009; 4: 62.

27. Granero-Moltó F, Weis JA, Miga MI, Landis B, Myers TJ, O'Rear L, Longobardi L, Jansen ED, Mortlock DP, and Spagnoli A: Regenerative ef- fects of transplanted mesenchymal stem cells in fracture healing. Stem Cells 2009; 27: 1887.

28. Kawabata M, Imamura $T$, and Miyazono $\mathrm{K}$ : Signal transduction by bone morphogenetic proteins. Cytokine Growth Factor Rev 1998; 9: 49.

29. Chen G, Deng $C$, and Li YP: TGF- $\beta$ and BMP signaling in osteoblast differentiation and bone formation. Int J Biol Sci 2012; 8: 272.

30. Bandyopadhyay A, Tsuji K, Cox K, Harfe BD, Rosen V, and Tabin CJ: Genetic analysis of the roles of BMP2, BMP4, and BMP7 in limb patterning and skeletogenesis. PLoS Genet 2006; 2: e216.

31. Tsuji K, Bandyopadhyay A, Harfe BD, Cox K, Kakar S, Gerstenfeld L, Einhorn T, Tabin CJ, and Rosen V: BMP2 activity, although dispensable for bone formation, is required for the initiation of fracture healing. Nat Genet 2006; 38: 1424.

32. Tsuji K, Cox K, Bandyopadhyay A, Harfe BD, Tabin $\mathrm{CJ}$, and Rosen V: BMP4 is dispensable for skeletogenesis and fracture-healing in the limb. $J$ Bone Joint Surg Am 2008; 90 Suppl 1: 14.

33. Ling L, Nurcombe V, and Cool SM: Wnt signaling controls the fate of mesenchymal stem cells. Gene 2009; 433: 1.

34. Boland GM, Perkins G, Hall DJ, and Tuan RS: Wnt 3a promotes proliferation and suppresses osteogenic differentiation of adult human mesenchymal stem cells. J Cell Biochem 2004; 93: 1210.

35. Qiu W, Andersen TE, Bollerslev J, Mandrup S, Abdallah BM, and Kassem M: Patients with high bone mass phenotype exhibit enhanced osteoblast differentiation and inhibition of adipogenesis of human mesenchymal stem cells. J Bone Miner Res 2007; 22: 1720.

36. De Boer J, Wang HJ, and Van Blitterswijk C: Effects of Wnt signaling on proliferation and differentiation of human mesenchymal stem cells. Tissue Eng 2004; 10: 393.

37. Baksh D and Tuan RS: Canonical and noncanonical Wnts differentially affect the development potential of primary isolate of human bone marrow mesenchymal stem cells. J Cell Physiol 2007; 212: 817

38. Cawthorn WP, Bree AJ, Yao Y, Du B, Hemati N, Martinez-Santibañez $G$, and MacDougald $O A$ : Wnt6, Wnt10a and Wnt10b inhibit adipogenesis and stimulate osteoblastogenesis through a $\beta$ catenin-dependent mechanism. Bone 2012; 50: 477.

39. Watt FM, Estrach S, and Ambler CA: Epidermal Notch signalling: differentiation, cancer and adhesion. Curr Opin Cell Biol 2008; 20: 171.

40. Engin F, Yao Z, Yang T, Zhou G, Bertin T, Jiang MM, Chen Y, Wang L, Zheng H, Sutton RE, Boyce $\mathrm{BF}$, and Lee B: Dimorphic effects of Notch signaling in bone homeostasis. Nat Med 2008; 14: 299. 
41. Deregowski V, Gazzerro E, Priest L, Rydziel S, and Canalis E: Notch 1 overexpression inhibits osteoblastogenesis by suppressing Wnt/beta-catenin but not bone morphogenetic protein signaling J Biol Chem 2006; 281: 6203.

42. Sen MK and Miclau T: Autologous iliac crest bone graft: should it still be the gold standard for treating nonunions? Injury 2007; 38 Suppl 1: S75.

43. Kasten P, Vogel J, Geiger F, Niemeyer P, Luginbühl $R$, and Szalay K: The effect of platelet-rich plasma on healing in critical-size long-bone defects. Biomaterials 2008; 29: 3983.

44. Schmidmaier G, Herrmann S, Green J, Weber T, Scharfenberger A, Haas NP, and Wildemann B: Quantitative assessment of growth factors in reaming aspirate, iliac crest, and platelet preparation. Bone 2006; 39: 1156

45. Veillette CJ and McKee MD: Growth factorsBMPs, DBMs, and buffy coat products: are there any proven differences amongst them? Injury 2007; 38 Suppl 1: S38.

46. Betz $O B$, Betz VM, Nazarian A, Egermann $M$, Gerstenfeld LC, Einhorn TA, Vrahas MS, Bouxsein $\mathrm{ML}$, and Evans $\mathrm{CH}$ : Delayed administration of adenoviral BMP-2 vector improves the formation of bone in osseous defects. Gene Ther 2007; 14: 1039 .
47. Wildemann B, Kandziora F, Krummrey G, Palasdies $N$, Haas NP, Raschke $M$, and Schmidmaier G: Local and controlled release of growth factors (combination of IGF-I and TGF-beta I, and BMP-2 alone) from a polylactide coating of titanium implants does not lead to ectopic bone formation in sheep muscle. J Control Release 2004; 95: 249.

48. Kakar S, Einhorn TA, Vora S, Miara LJ, Hon G, Wigner NA, Toben D, Jacobsen KA, Al-Sebaei MO, Song M, Trackman PC, Morgan EF, Gerstenfeld LC, and Barnes GL: Enhanced chondrogenesis and Wnt signaling in PTH-treated fractures. J Bone Miner Res 2007; 22: 1903

49. Manabe T, Mori S, Mashiba T, Kaji Y, Iwata K, Komatsubara S, Seki A, Sun YX, and Yamamoto T: Human parathyroid hormone (1-34) accelerates natural fracture healing process in the femoral osteotomy model of cynomolgus monkeys. Bone 2007; 40: 1475.

50. Komatsu DE, Mary MN, Schroeder RJ, Robling $\mathrm{AG}$, Turner $\mathrm{CH}$, and Warden SJ: Modulation of Wnt signaling influences fracture repair. J Orthop Res 2010; 28: 928.

51. Tian X, Jee WS, Li X, Paszty C, and Ke HZ: Sclerostin antibody increases bone mass by stimulating bone formation and inhibiting bone resorption in a hindlimb-immobilization rat model. Bone 2011; 48: 197.
52. Myers TJ, Yan Y, Granero-Molto F, Weis JA, Longobardi L, Li T, Li Y, Contaldo C, Ozkan H, and Spagnoli A: Systemically delivered insulin-like growth factor-I enhances mesenchymal stem celldependent fracture healing. Growth Factors 2012 30: 230.

53. Hernigou P, Poignard A, Beaujean F, and Rouard $\mathrm{H}$ : Percutaneous autologous bone-marrow grafting for nonunions. Influence of the number and concentration of progenitor cells. J Bone Joint Surg Am 2005; 87: 1430

54. Bruder SP, Kraus KH, Goldberg VM, and Kadiyala $S$ : The effect of implants loaded with autologous mesenchymal stem cells on the healing of canine segmental bone defects. J Bone Joint Surg Am 1998; 80: 985.

55. Janicki P, Kasten P, Kleinschmidt K, Luginbuehl $R$ and Richter W: Chondrogenic pre-induction of human mesenchymal stem cells on beta-TCP: enhanced bone quality by endochondral heterotopic bone formation. Acta Biomater 2010; 6: 3292.

56. Ushiku C, Adams DJ, Jiang X, Wang L, and Rowe DW: Long bone fracture repair in mice harboring GFP reporters for cells within the osteoblastic lineage. J Orthop Res 2010; 28: 1338.

57. Schraufstatter IU, Discipio RG, and Khaldoyanidi S: Mesenchymal stem cells and their microenvironment. Front Biosci 2011; 16: 2271. 